\title{
Density dependence of the extinction coefficient of a dispersion of spherical metal particles*)
}

\author{
E. Fucile, P. Denti, R. Saija, F. Borghese, and O. I. Sindoni ${ }^{1}$ ) \\ Università di Messina, Istituto di Struttura della Materia, S. Agata-Messina, Italy \\ $\left.{ }^{1}\right)$ Chemical Research Development and Engineering Center, Aberdeen P.G., Maryland, USA
}

\begin{abstract}
The multiple scattering processes are assumed to have a negligible effect on the optical properties of a low-density dispersion of scatterers. Since the range of low-density is known only by estimates [1], we make an attempt to assess down to what density the extinction coefficient of a dispersion of spherical scatterers is influenced by the multiple scattering effects. To this end, the scatterers of the dispersion are considered as aggregated in pairs (even if they are not in contact). The field scattered by each pair is then calculated by means of a theory we developed in the last few years especially to deal with aggregates of spherical scatterers [2]. Of course, the field scattered by each pair depends on its orientation with respect to the incoming field, as well as on the separation of the component spheres. However, by means of the theory cited above, we are able to sum analytically over the orientations to get the field scattered, on the average, by the dispersion of pairs. As the resulting field is still a function of the separation of the
\end{abstract}

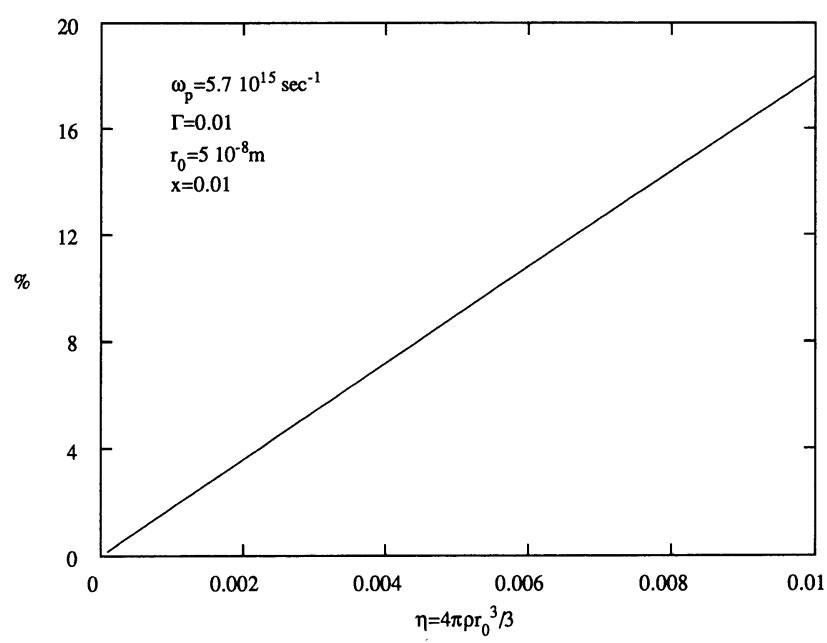

Fig. 1 spheres composing the pair, we make an average over the separation using as a weight the pair correlation function for a dispersion with the density we are dealing with. As a result, we get forward-scattering amplitude of each sphere dressed by the effect of the two-body multiplescattering processes produced by the surrounding particles. The calculation of the corrected optical properties of the dispersion is now a matter of introducing the dressed amplitude into the customary formulas [3]. As an example, we shows in Fig. 1 the correction to the extinction coefficient of a dispersion of spherical metal particles as a function of the packing fraction. It is quite evident that the correction due to the two-body multiple-scattering processes has a considerable effect, even when the density of the dispersion is rather low.

Key words: Refractive index; scattering; propagation in non-homogeneous media

\section{References}

1. Bayvel LP, Jones AR (1981) Electromagnetic Scattering and Its Applications. Applied Science, London

2. Borghese F, Denti P, Saija R, Toscano G, Sindoni OIK (1984) Aerosol Sci Technol 3:227

3. Newton RG (1966) Scattering theory of waves and particles. Mc Graw-Hill, New York

Authors' address:

Dr. F. Borghese

Università di Messina

Istituto di Struttura della Materia

Salita Sperone 31

98166 S. Agata-Messina, Italy

*) Based on work supported inpart by the U. S. Army European Research Office through Contract DAJA45-86-C-0003 and in part by the Consiglio Nazionale delle Ricerche through the Gruppo Nazionale Strutura della Materia. 